\title{
20. Gene Dosage Effect on Rh Blood Group Antigens observed in Erythrocytes Heterozygous for $\mathrm{Rh}^{-29}(\overline{\bar{r}}, \ldots)$ Gene
}

\author{
By Hayato Hasekura, *) Masao Ota,*) Hirofumi Fukushima,*) \\ Isamu YonemurA,*) and Takahira IsHIMoRI**) \\ (Communicated by Shoei IsekI, M. J. A., Feb. 12, 1986)
}

Erythrocytes with different Rh blood group phenotypes differ in their reactivity against various $\mathrm{Rh}$ antibodies. This difference has been attributed to gene dosage effect or disturbing effect by other antigen constructions. ${ }^{1)}$

We discovered earlier, the first $\mathrm{Rh}:-29,38\left(\overline{\overline{\mathrm{r}}}, \mathrm{-}----_{--}\right)$propositus, a homozygote of the amorph gene $\mathrm{R}^{-29}(\overline{\overline{\mathrm{r}}},---)$, together with several heterozygotes in his family. ${ }^{2)}$ Comparison of these heterozygotes with homozygotes should provide critical samples in interpreting the difference of agglutination reactions, as the $\mathrm{Rh}$ antigens on these cells are products of single genes.

This report presents the data of comparative titration using classic agglutination reaction expressed in scores.

Materials and methods. Erythrocytes. The family members of H. A., the original $\mathrm{Rh}:-29,38(\overline{\overline{\mathrm{r}}} \mathrm{h},---/---)$, including known heterozygotes of $\mathrm{R}^{-29}(\overline{\overline{\mathrm{r}}},---)$

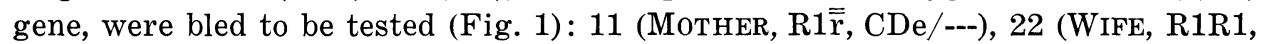

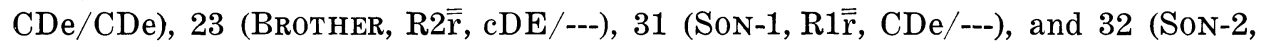
$\mathrm{R} 1 \overline{\overline{\mathrm{r}}}, \mathrm{CDe} /---)$. Also, family members of a disputed paternity case were included in the experiment (Fig. 2), where Child-3 (a three-year-old girl, $\mathrm{R} 2 \overline{\overline{\mathbf{r}}}, \mathrm{cDE} /---)$ was annulled by Father (R1) $\overline{\mathrm{r}}$, $\mathrm{CDe} /---)$; she was excluded of legitimacy at the first trial on the basis that R1R1 (CCDee) man could not have an R2R2 (ccDEE) child. The second instance extended blood group tests to Child-1 (R2 $\overline{\bar{r}}, \mathrm{cDE} /---)$ and Child-2 (R1R2, CDe/cDE) both undisputed on their legitimacy; Child-3 was then confirmed to be a legitimate, as Child-1, her brother, revealed the same Rh phenotype as hers.

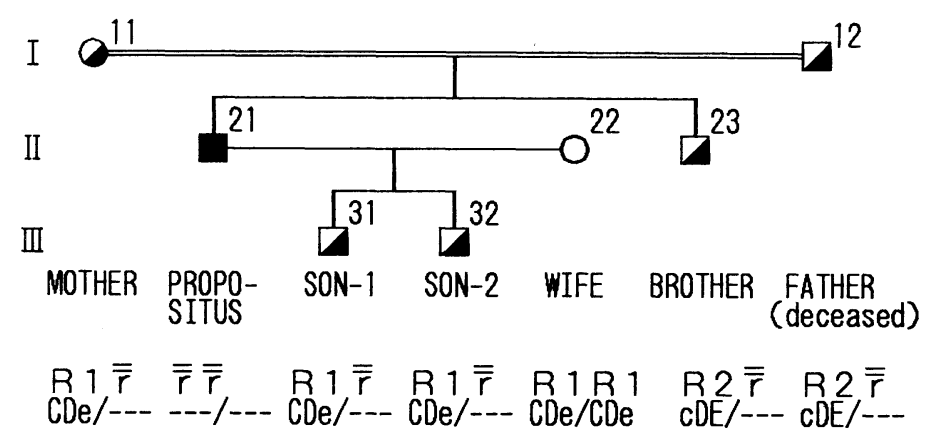

Fig. 1. Pedigree of H. A., the original Rh: $-29,38(\overline{\bar{r} h},--/--)$.

\footnotetext{
*) Department of Legal Medicine, Shinshu University School of Medicine, Matsumoto, 390 Japan.

**) Funabashi Red Cross Blood Center, Funabashi, 274 Japan.
} 

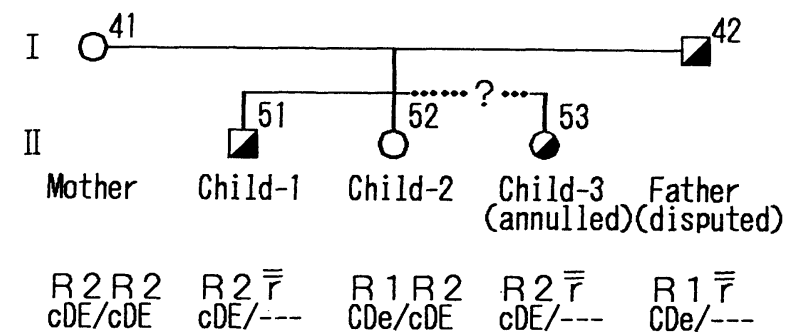

Fig. 2. Pedigree of a disputed paternity case where $\mathrm{Rh}^{-29}(\overline{\overline{\mathrm{r}}},--)$ gene was involved. Child-3, first excluded of legitimacy, was confirmed through the additional tests including Child-1, her brother.

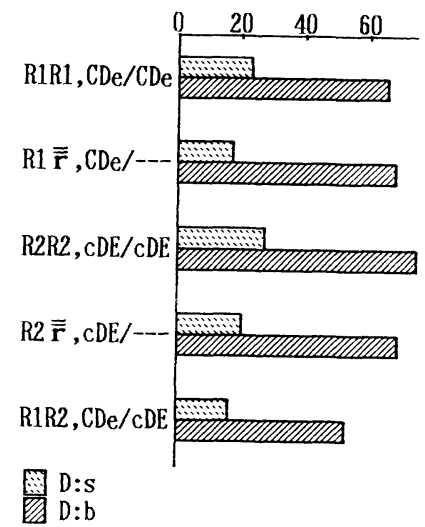

Fig. 3. Average Rh 1(Rho, D) antigen strength quantitated in agglutination scores. D:s, saline agglutination; $\mathrm{D}: \mathrm{b}$, bromelin agglutination. No dosage effect was observed.

Titration. Rh blood grouping sera (Ortho Diagnostics) were serially diluted with physiological saline to be delivered in small test tubes $0.04 \mathrm{ml}$ each, added with $0.04 \mathrm{ml}$ suspension of $5 \%$ erythrocyte in saline to make two sets, one for saline and the other for bromelin tests; for saline tests, tubes were centrifuged after 20 min incubation in $37^{\circ} \mathrm{C}$ bath to be read for agglutination. For bromelin tests, $0.04 \mathrm{ml}$ of $0.5 \%$ bromelin solution was added to each tube with antiserumerythrocyte mixture; after $20 \mathrm{~min}$ in $37^{\circ} \mathrm{C}$, centrifugation and recording followed. Strength of agglutination was scored according to Marsh, ${ }^{3)} 4^{+}: 12,3^{+}: 10,2^{+}: 8$, $1^{+}: 5, \mathrm{w}^{+}: 2,-: 0$. Agglutination scores were summed up to represent the agglutinability of erythrocytes with $\mathrm{Rh}$ antisera.

Results and discussion. Agglutination scores of different types of erythrocytes are compared in Table I and Figs. 3-5.

Agglutination scores against anti-Rh 1 (Rho, D) revealed considerable variations among different erythrocytes (Fig. 3); in average, however, $\mathrm{R}^{-29}(\overline{\overline{\mathrm{r}}},---)$ heterozygotes did not show decreased antigen reactivity compared with ordinary types of cells (Table I), although the sample numbers were too small for statistical considerations. Incidentally, heterozygotes in the disputed family (42: Father, 51: Child-1 and 52: Child-2, Fig. 2) did not reveal an increase in agglutination reactivity to anti-Rh 1 (Rho, D) serum, excluding the possible participation of $\mathrm{R}^{1,-17}\left(\overline{\mathrm{R}}^{0},-\mathrm{D}-\right)$ gene.

Agglutination scores against anti-Rh 2 ( $\left.\mathrm{rh}^{\prime}, \mathrm{C}\right)$ and anti-Rh 5 (hr', e) showed that $\mathrm{R}^{-29}(\overline{\bar{r}},---)$ heterozygous cells possess decreased antigen reactivity compared 
Table I. Agglutination scores of erythrocytes with $\mathrm{Rh}$ antisera

\begin{tabular}{|c|c|c|c|c|c|c|c|c|c|c|}
\hline \multirow{2}{*}{$\begin{array}{l}\text { Antisera } \\
\text { Methods }\end{array}$} & \multicolumn{2}{|c|}{ Rh 1(Rho, D) } & \multicolumn{2}{|c|}{$\mathrm{Rh} 2\left(\mathrm{rh}^{\prime}, \mathrm{C}\right)$} & \multicolumn{2}{|c|}{$\mathrm{Rh} 5\left(\mathrm{hr} \mathrm{r}^{\prime \prime}, \mathrm{e}\right)$} & \multicolumn{2}{|c|}{$\mathrm{Rh} 3\left(\mathrm{rh}^{\prime \prime}, \mathrm{E}\right)$} & \multicolumn{2}{|c|}{$\mathrm{Rh} 4\left(\mathrm{hr}^{\prime}, \mathrm{c}\right)$} \\
\hline & Sal. & Brom. & Sal. & Brom. & Sal. & Brom. & Sal. & Brom. & Sal. & Brom. \\
\hline \multicolumn{11}{|l|}{$\mathrm{Rh}$ genotype } \\
\hline $\mathrm{R} 1 \mathrm{R} 1, \mathrm{CDe} / \mathrm{CDe}$ & 23.0 & 65.7 & 33.7 & 62.0 & 30.7 & 64.0 & $\underline{0}$ & $\underline{0}$ & $\underline{0}$ & $\underline{0}$ \\
\hline 22 WIFE & 23 & 65 & 33 & 46 & 17 & 63 & 0 & 0 & 0 & 0 \\
\hline Normal & 29 & 81 & 30 & 61 & 22 & 67 & 0 & 0 & 0 & 0 \\
\hline Normal & 17 & 51 & 38 & 79 & 53 & 62 & 0 & 0 & 0 & 0 \\
\hline $\mathrm{R} 1 \overline{\overline{\mathrm{r}}}, \mathrm{CDe} /--$ & 17.3 & 68.3 & 19.0 & 51.5 & 16.8 & 44.2 & $\underline{0}$ & $\underline{0}$ & $\underline{0}$ & $\underline{0}$ \\
\hline 11 MOTHER & 20 & 66 & 19 & 50 & 12 & 45 & 0 & 0 & 0 & 0 \\
\hline 31 SoN-1 & 17 & 68 & 19 & 50 & 12 & 47 & 0 & 0 & 0 & 0 \\
\hline 32 SoN-2 & 17 & 68 & 19 & 53 & 12 & 47 & 0 & 0 & 0 & 0 \\
\hline 42 FATHER & 15 & 71 & 19 & 53 & 31 & 38 & 0 & 0 & 0 & 0 \\
\hline $\mathrm{R} 2 \mathrm{R} 2, \mathrm{cDE} / \mathrm{cDE}$ & 27.5 & 74.5 & $\underline{0}$ & $\underline{0}$ & $\underline{0}$ & $\underline{0}$ & 48.0 & 72.0 & 27.0 & 85.5 \\
\hline 41 MOTHER & 32 & 88 & 0 & 0 & 0 & 0 & 48 & 66 & 27 & 81 \\
\hline Normal & 23 & 61 & 0 & 0 & 0 & 0 & 48 & 78 & 27 & 90 \\
\hline $\mathrm{R} 2 \overline{\overline{\mathrm{r}}}, \mathrm{cDE} /--$ & 20.3 & 68.7 & $\underline{0}$ & $\underline{0}$ & $\underline{0}$ & $\underline{0}$ & 24.3 & 57.0 & 20.3 & 70.7 \\
\hline 23 BROTHER & 17 & 64 & 0 & 0 & 0 & 0 & 45 & 67 & 19 & 77 \\
\hline 51 CHILD-1 & 22 & 71 & 0 & 0 & 0 & 0 & 36 & 49 & 20 & 66 \\
\hline 53 CHILD-3 & 22 & 71 & 0 & 0 & 0 & 0 & 33 & 55 & 22 & 69 \\
\hline $\mathrm{R} 1 \mathrm{R} 2, \mathrm{CDe} / \mathrm{cDE}$ & 16.5 & 52.5 & 23.0 & 49.5 & 19.7 & 37.0 & 31.5 & 45.0 & 15.5 & 62.5 \\
\hline 52 CHILD-2 & 13 & 55 & 23 & 52 & 28 & 40 & 28 & 37 & 17 & 64 \\
\hline Normal & 20 & 50 & 23 & 47 & 8 & 43 & 35 & 53 & 14 & 61 \\
\hline Normal & & & & & 23 & 28 & & & & \\
\hline
\end{tabular}

Underlined figures show average scores of the genotype.

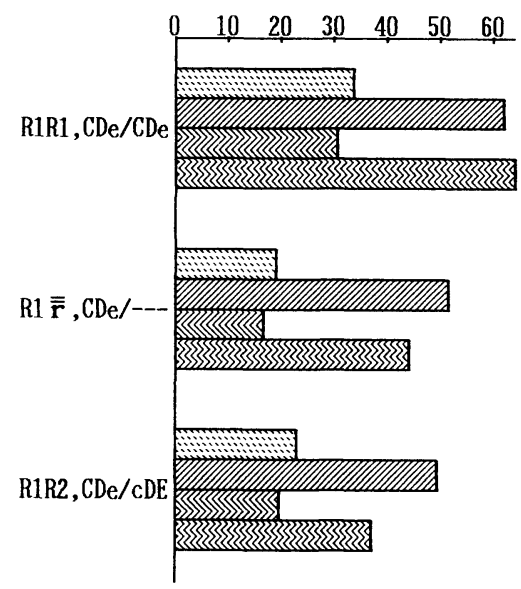

Fig. 4
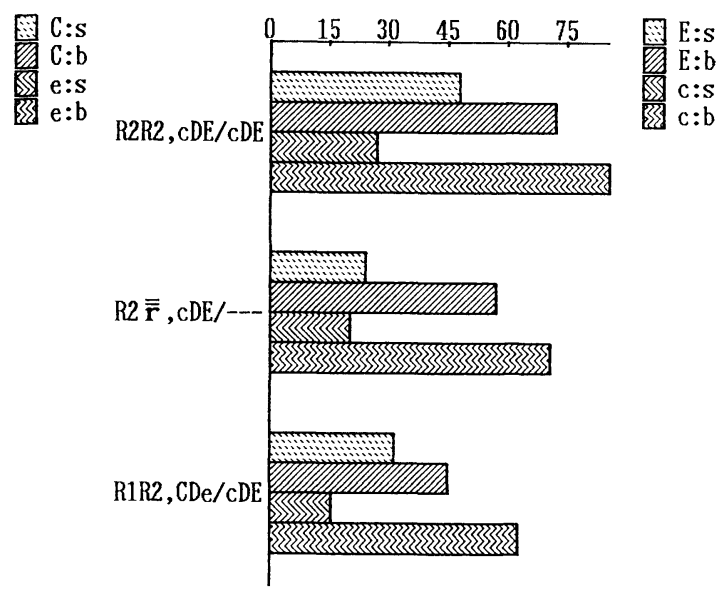

Fig. 5

Figs. 4-5. 4: Average $\mathrm{Rh} 2\left(\mathrm{rh}^{\prime}, \mathrm{C}\right)$ and $\mathrm{Rh} 5\left(\mathrm{hr}^{\prime \prime}, \mathrm{e}\right)$ antigen strength quantitated in agglutination scores. s, saline agglutination; b, bromelin agglutination. Suggestive dosage effect was observed. 5: Average Rh 3(rh", E) and Rh 4(hr', c) antigen strength quantitated in agglutination scores. $\mathrm{s}$, saline agglutination, $\mathrm{b}$, bromelin agglutination. Suggestive dosage effect was observed. 
[Vol. 62(B),

with R1R1 (CDe/CDe) cells, thus resembling R1R2 (CDe/cDE) heterozygotes (Figs. 4-5, Table I).

Also anti-Rh $3\left(\mathrm{rh}^{\prime \prime}, \mathrm{E}\right)$ and anti-Rh $4\left(\mathrm{hr}^{\prime}, \mathrm{c}\right)$ agglutinated $\mathrm{R}^{-29}(\overline{\overline{\mathrm{r}}},---)$ heterozygotes weaker than homozygous R2R2 (cDE/cDE) cells; reactivity of heterozygous R1R2 (CDe/cDE) cells was still weaker probably due to some disturbing effect on anti-Rh 1 (Rho, D) antibody reaction.

These results would suggest that gene dosage effect works on $\mathrm{Rh} 2-5$ ( $\mathrm{rh}^{\prime}, \mathrm{C}$; $\left.\mathrm{rh}^{\prime \prime}, \mathrm{E} ; \mathrm{hr}^{\prime}, \mathrm{c} ; \mathrm{hr}^{\prime \prime}, \mathrm{e}\right)$ antigens but not on Rh 1 (Rho, D) antigen; in other words, $\mathrm{Rh} 2-5\left(\mathrm{rh}^{\prime}, \mathrm{C} ; \mathrm{rh}^{\prime \prime}, \mathrm{E} ; \mathrm{hr}^{\prime}, \mathrm{c}\right.$; $\left.\mathrm{hr^{ \prime \prime }}, \mathrm{e}\right)$ antigens are incompletely dominant traits while $\mathrm{Rh} 1$ (Rho, D) antigen is completely dominant.

Also in the German family including the second and the third case of $\overline{\mathrm{r}} \mathrm{h}$ $(---/--)$ individuals, heterozygotes of the $\mathrm{R}^{-29}(\overline{\overline{\mathrm{r}}},---)$ gene revealed the single dose antigen activity in agreement with the present study. ${ }^{4}$

Summary. $R^{-29}(\overline{\bar{r}},---)$ heterozygous erythrocytes were titrated against $R h$ blood grouping antisera in comparison with ordinary cells. Gene dosage effect was suggested to work on $\mathrm{Rh} 2-5\left(\mathrm{rh}^{\prime}, \mathrm{C} ; \mathrm{rh}^{\prime \prime}, \mathrm{E} ; \mathrm{hr}^{\prime}, \mathrm{c} ; \mathrm{hr} \mathbf{r}^{\prime \prime}, \mathrm{e}\right)$ antigens but not on Rh 1 (Rho, D) antigen.

\section{References}

1) R. R. Race and R. Sanger: Blood Groups in Man. 6th ed., Blackwell (Oxford), pp. 232-236 (1975).

2) T. Ishimori and H. Hasekura: A case of a Japanese blood with no detectable Rh blood group antigen. Discovery of a homozygote of deleted chromosomes or silent Rh alleles. Proc. Japan Acad., 42, 658-660 (1966).

3) W. L. Marsh: Scoring of hemagglutination rections. Transfusion, 12, 352-353 (1972).

4) S. Seidl, W. Spielmann, and H. Martin: Two siblings with Rh null disease. Vox Sang., 23, 182-189 (1972). 